\title{
Anthropometric and Biochemical Markers of Insulin Resistance
}

\author{
Sneha $S^{1}$, M Sudhakar Rao ${ }^{2}$, Sudha Vidyasagar ${ }^{3}$ \\ ${ }^{1}$ Department of Medicine, KMC Manipal , Manipal University \\ ${ }^{2}$ Department of Cardiology, KMC Manipal , Manipal University \\ ${ }^{3}$ Department of Medicine, KMC Manipal , Manipal University
}

\begin{abstract}
Insulin resistance is associated with obesity, impaired glucose tolerance (IGT) and type 2 diabetes mellitus, predisposing to adverse cardiovascular outcomes and cerebrovascular disease. This makes it important to identify persons with increased insulin resistance as they are at high risk of vascular problems and related morbidity and mortality. This study was done to assess noninvasive markers of insulin resistance used in routine clinical practice such as anthropometry, lipid profile and hsCRP and to determine their correlation with insulin resistance. A cross- sectional study including 90 subjects was conducted. Each participant underwent anthropometric assessment including BMI, Waist and Hip circumference measurements, fasting insulin, fasting glucose, fasting lipid profile and hsCRP measurements. Statistical Analysis was done using SPSS version 15 and results analyzed by spearman's correlation coefficient. Linear regression analysis was done to find the relation between HOMA-IR, anthropometry and lipid profile.BMI, waist circumference and serum triglyceride levels showed significant correlation with log HOMA-IR indicating that they are significant noninvasive markers of insulin resistance. Hence simple anthropometry and routine biochemical investigations done as standard care can be used to identify patients with insulin resistance. Appropriate remedial life style and other measures can be used in these patients to avoid adverse vascular outcomes.
\end{abstract}

Keywords: Insulin resistance, HOMA-IR, anthropometric measurements for insulin resistance, HOMA-IR and hsCRP, HOMA-IR and lipid profile

\section{Introduction:}

Insulin resistance is a strong predictor of cardiovascular risk and current focus of research. It is associated with obesity, hypertension, coronary artery disease, dyslipidemias and a cluster of metabolic and cardiovascular abnormalities that define the metabolic syndrome. Insulin resistance is difficult to diagnose clinically, surrogate indices are needed for recognition of insulin resistance. This study was done to determine noninvasive markers of insulin resistance and to determine their correlation with insulin resistance.

\section{Aims and Objectives}

To determine the correlation of Homeostasis Model Assessment Insulin Resistance (HOMAIR) with surrogate markers such as-

- Anthropometric measurements -

Body mass index (BMI)

Waist Circumference (WC)

Waist Hip Ratio (WHR)

- Biochemical markers -

Highly sensitive C Reactive Protein(hsCRP)

High Density Lipoprotein (HDL)

Triglyceride/ HDL (TG/HDL) ratio

\section{Methodology}

A cross- sectional study including 90 subjects was conducted. Each participant underwent -
Anthropometric assessment which included BMI, Waist and Hip circumferences, fasting insulin, fasting glucose, hsCRP estimation and fasting lipid profile.

BMI was calculated using the formula- $\mathrm{BMI}=$ Weight $(\mathrm{Kg}) /$ $[\operatorname{Height}(\mathrm{m})]^{2}$

Waist circumference: A plastic measuring tape was applied tightly applied to the skin surface so that the tape was taut but not tight. Waist circumference was measured midway between inferior margin of ribs and the superior border of iliac crest.

Hip circumference: Widest diameter over the greater trochanters was measured while the subjects were standing with their heels together.

Waist-Hip ratio: It was calculated using the formula-

$$
\text { W-H ratio }=\frac{\text { Waist circumference }}{\text { Hip circumference }}
$$

Fasting insulin was estimated using the chemiluminescence method

hs-CRP was measured using the Immunoturbidimetric method.

Estimation of FBS was done by hexokinase method

Total cholesterol was estimated by CHOD-POD enzymatic method.

Estimation of HDL cholesterol as done by direct homogenous method. 


\section{International Journal of Science and Research (IJSR) \\ ISSN (Online): 2319-7064}

Index Copernicus Value (2013): 6.14 | Impact Factor (2014): 5.611

LDL was calculated using the formula - Total cholesterol $($ HDL-C + Triglycerides $)=$ LDL Cholesterol.

HOMA-IR was calculated using the standard formula [Fasting Glucose (mmol/L) X insulin (mu/L)]

All the biochemical tests were done using ROCHE diagnostics machine, Germany.

Statistical analysis was done using SPSS version 15 and results analysed by spearman's correlation coefficient.

Linear regression analysis was done to find the relation between HOMA-IR, anthropometry and lipid profile.

Log transformation of HOMA-IR was done as data was skewed. $\mathrm{p}$ value $\leq 0.05$ was taken as significant.

\section{Results}

Table 4.1: Patient Characteristics

\begin{tabular}{|c|c|}
\hline Patient Characteristic & $\mathbf{n = 9 0}$ \\
\hline MEAN AGE & $45 \pm 11.51$ years \\
\hline MALES & 52 \\
\hline FEMALES & 38 \\
\hline DIABETICS & $\mathbf{2 1}$ \\
\hline NON DIABETICS & $\mathbf{6 9}$ \\
\hline
\end{tabular}

Table 4.2

\begin{tabular}{|c|c|c|}
\hline$Q 1$ & MEDIAN HOMA-IR & $Q 3$ \\
\hline 1.66 & $\mathbf{3 . 0 4}$ & 5.79 \\
\hline
\end{tabular}

Median HOMA-IR was 3.04 in the study population

Table 4.3: Correlation of Homa-IR With Anthropometric Measurements

\begin{tabular}{|c|c|c|}
\hline HOMA-IR VS & Spearman's Coefficient & $p$ value \\
\hline Body Mass Index & $\mathbf{0 . 6 0 7}$ & $\mathbf{0 . 0 0 0} *$ \\
\hline Waist Circumference & $\mathbf{0 . 6 1 3}$ & $\mathbf{0 . 0 0 0} *$ \\
\hline Waist Hip Ratio & $\mathbf{0 . 4 7 7}$ & $\mathbf{0 . 0 0 0} *$ \\
\hline
\end{tabular}

Table 4.4: Correlation of Homa-IR With Lipid Profile and hsCRP

\begin{tabular}{|c|c|c|}
\hline HOMA-IR V/S & SPEARMAN'S COEFFICIENT & $\mathrm{p}$ value \\
\hline TG/HDL & 0.597 & $0.000^{*}$ \\
\hline HDL & -0.433 & $0.000^{*}$ \\
\hline hsCRP & 0.474 & $0.000^{*}$ \\
\hline
\end{tabular}

$*_{p}<0.05$ - significant

Since the correlation was highly significant, we did a regression analysis to find out the variable with maximum impact.

Table 4.5: Multiple Linear Regression Analysis Coefficients

\begin{tabular}{|c|c|c|c|c|}
\hline Model & $\begin{array}{c}\text { Standard } \\
\text { coefficients (beta) }\end{array}$ & $t$ & Sig & $\begin{array}{c}\text { 95\% confidence } \\
\text { interval }\end{array}$ \\
\hline BMI & 0.237 & 2.089 & $\mathbf{0 . 0 4 0} *$ & $0.001-0.058$ \\
\hline $\begin{array}{c}\text { Waist } \\
\text { circumference }\end{array}$ & 0.325 & 2.214 & $\mathbf{0 . 0 3 0} *$ & $0.002-0.035$ \\
\hline Waist Hip ratio & 0.030 & 0.276 & 0.783 & $-2.2625-3.471$ \\
\hline HDL & -0.092 & -1.032 & 0.305 & $-0.020-0.006$ \\
\hline Triglyceride & 0.302 & 3.545 & $\mathbf{0 . 0 0 1} *$ & $0.001-0.004$ \\
\hline
\end{tabular} $\begin{aligned} & \text { a dependent variable logHOMA-IR } \\
& { }_{p}<0.05-\text { significant }\end{aligned}$

\section{Discussion}

A simple clinical test cannot predict insulin resistance, hence surrogate markers are needed for timely detection of it. We assessed how effectively different anthropometric measurements and biochemical markers used in clinical practice can predict insulin sensitivity.

In our study, we found that the median HOMA - IR was $>2.5$ suggesting increased prevalence of insulin resistance among the study population.

Common and simple anthropometric measurements like BMI, waist circumference and waist hip ratio (visceral obesity) were found to have significant positive correlation with HOMA-IR

A significant positive correlation was also established between TG/HDL and HOMA-IR suggesting higher TG levels depict insulin resistance.

A significant negative correlation was found between HOMA-IR and HDL emphasizing that insulin sensitivity decreases with HDL.

TG and TG/HDL ratio can be used to identify insulin resistant individuals is shown in this study. These parameters were shown to have the same degree of specificity and sensitivity as plasma insulin ${ }^{3}$.

hsCRP and HOMA-IR also showed a significant positive correlation $(r=0.474)$ emphasizing that insulin resistance is an inflammatory state and higher the hsCRP levels, higher the insulin resistance is.

Inflammation and insulin resistance have shown to be forerunners of diabetes and cardiovascular diseases. ${ }^{4}$ Multiple linear regression analysis showed following parameters to have statistical significant relation with $\log$ HOMA -IR:

- BMI $(\mathrm{p}=0.04,95 \%$ CI $0.001-0.058)$, WC $(\mathrm{p}=0.030$ 95\%CI $0.002-0.035), \mathrm{TG}(\mathrm{p}=0.001,95 \%$ CI $0.001-0.004)$ These could be surrogate clinical markers of insulin resistance ${ }^{5}$.

The coefficient of determination (R2) was observed to be $52 \%$ suggesting that these factors play a major role in identifying insulin resistance

\section{Conclusions}

- Anthropometric measurements, TG/HDL ratio, low HDL levels and hsCRP levels can serve as surrogate markers of insulin resistance.

- BMI, waist circumference and serum triglyceride levels showed significant correlation with log HOMA-IR indicating that they are significant and better noninvasive markers of insulin resistance. Hence simple anthropometry and routine biochemical investigations done as standard care can be used to identify patients with insulin resistance.

\section{Volume 5 Issue 1 January 2016}


- Appropriate remedial lifestyle and other measures can be used in these patients to avoid adverse vascular outcomes

\section{References}

[1] Muniyappa R, Lee S, Chen H, Quon MJ. Current approaches for assessing insulin sensitivity and resistance in vivo: advantages, limitations, and appropriate usage. Am J Physiol Endocrinol Metab. 2008 Jan;294(1):E1526.

[2] Kahn SE, Prigeon RL, Schwartz RS, Fujimoto WY, Knopp RH, Brunzell JD, et al. Obesity, body fat distribution, insulin sensitivity and Islet betacell function as explanations for metabolic diversity. J Nutr. 2001;131(2):354S-60S

[3] McLaughlin T, Reaven G, Abbasi F, Lamendola C, Saad M, Waters D, et al. Is There a Simple Way to Identify Insulin-Resistant Individuals at Increased Risk of Cardiovascular Disease? American Journal of Cardiology.96(3):399-404

[4] Rhee EJ, Kim YC, Lee WY, Jung CH, Sung KC, Ryu SH, et al. Comparison of insulin resistance and serum highsensitivity C-reactive protein levels according to the fasting blood glucose subgroups divided by the newly recommended criteria for fasting hyperglycemia in 10059 healthy Koreans. Metabolism. 2006 Feb;55(2):183-7

[5] Anan F, Takahashi N, Nakagawa M, Ooie T, Saikawa T, Yoshimatsu H. High-sensitivity C-reactive protein is associated with insulin resistance and cardiovascular autonomic dysfunction in type 2 diabetic patients. Metabolism. 2005 Apr;54(4):552-8 